\title{
T-cell Receptor Excision Circle
}

National Cancer Institute

\section{Source}

National Cancer Institute. T-cell Receptor Excision Circle. NCI Thesaurus. Code C2561.

The reciprocal product of T-cell receptor (TCR) gene rearrang ement. Following TCR gene rearrangement the T-cell receptor excision circle (TREC) is maintained in the cell as a non-replicating episome. With each cell division the TREC is segregated to a single daughter cell and becomes diluted in the population. Recent TCR rearrangement can be detected by a spike in the number of TREC and can be used to quantify thymic T-cell output. 